\title{
Erratum to: Western Purple-Faced Langurs (Semnopithecus vetulus nestor) Feed on Ripe and Ripening Fruit in Human-Modified Environments in Sri Lanka
}

\author{
Joanna M. Setchell
}

Published online: 12 July 2012

(C) Springer Science+Business Media, LLC 2012

\section{Erratum to: Int J Primatol (2012) 33(1): 40-72 \\ DOI 10.1007/s10764-011-9538-3}

\begin{abstract}
This notice clarifies the relationship between this publication and a previous publication by the same author. The purpose of this notice is to correct the literature and ensure its integrity.
\end{abstract}

Subsequent to the publication of this paper it has been brought to my attention that it overlaps substantially with a previous report by the same author (Dela 2007). The International Journal of Primatology participates in the Committee on Publication Ethics (COPE, http://publicationethics.org/) and acts in accordance with their guidelines relating to the integrity of the work submitted to, or published in, the journal. Following the COPE guidelines with regard to suspected redundant publication in a published article I examined the two papers and identified partial overlap, i.e., when authors present some new findings in an article that also contains a substantial amount of previously published information. I contacted the author to request an explanation. The reply was unsatisfactory, so I informed the author that I would need to publish a correction notice clarifying which aspects of the 2012 paper had been published previously. Here, I consulted the COPE guidelines on retraction and corrections. I invited the author to write this statement, but this offer was declined. I then drafted a notice and sent it to the author in an attempt to agree a form of wording that is clear and informative to readers and acceptable to all parties. Unfortunately, however, we were unable to agree on the need for a correction.

The purpose of this notice is to correct the literature and ensure its integrity.

The online version of the original article can be found at http://dx.doi.org/10.1007/s10764-011-9538-3.

J. M. Setchell (ه)

Department of Anthropology, Durham University, Durham, UK

e-mail: joanna.setchell@durham.ac.uk 


\section{Details of the Overlap}

The two papers make use of the same data set. This is acceptable, provided that it is clear what work was done previously or is repeated and what uses the data in a new way. However, this is not the case.

Comparison of the abstracts reveals that many of the basic findings are the same in the two papers; for example: the study species is highly frugivorous; the importance of jack fruit consumption; their dietary habits are highly flexible. This overlap is not made clear in the 2012 abstract.

The first paper (Dela 2007) is mentioned in the introduction. However, the conclusions are stated only briefly, and the relationship between the two studies is not made clear.

Much of the basic data presented in the 2007 paper are reanalyzed in the 2012 paper, with minor changes, such as the splitting of some categories into more detailed sub-categories (e.g., Fig. 12007 and Table I 2012). In some cases some of the same data are presented, but split into a table and a figure, resulting in different values.

In addition, the discussion of the 2012 paper begins with the conclusion of the the 2007 paper, but does not say so.

There is a consistent overstating of the originality of the findings in the 2012 paper, as many already appear in the 2007 paper.

\section{Clarification of the Relationship Between the Two Papers}

The author has clarified the relationship between the two papers, as follows: the 2007 study compared the use of seasonal items and mature leaves between two groups of Semnopithecus vetulus nestor. It does not discuss fruit quality, whole fruits, or propensity for frugivory. The 2012 paper shows that the study groups ate fruit significantly more than all foliage (including leaf stems as well as leaves), and that this held even when only whole fruit was considered.

In particular, the first paragraph of the discussion in the 2012 publication reads: "This study provides clear evidence that Semnopithecus vetulus nestor living in environments modified by humans and with abundant sources of cultivated fruits had adopted a frugivorous dietary strategy unlike that of any other colobine studied to date." The key phrase here is "unlike any other colobine." While other colobines have been shown to use fruits and to use fruits more than leaves, the 2012 paper demonstrates that Semnopithecus vetulus nestor uses fleshy whole fruit which is ripening or ripe. In contrast, the 2007 publication makes no distinction of fruit type and refers to fruits that include those used for seeds only.

\section{Conclusion}

To avoid redundant publication, the 2012 paper should have presented all previous conclusions in the introduction, and made the relationship between the analyses in the two papers clear to the reader. All repetition of previous analyses should have been removed from the results. This would leave a brief paper making the distinction 
between ripe or ripening fruits versus fruits eaten just for seeds. This is unlikely to have been acceptable for publication in this journal.

Joanna M. Setchell

\section{References}

Dela, J. (2007). Seasonal food use strategies of Semnopithecus vetulus nestor, at Panadura and Piliyandala, Sri Lanka. International Journal of Primatology, 28, 607-626.

Dela, J. (2012). Western purple-faced langurs (Semnopithecus vetulus nestor) feed on ripe and ripening fruit in human-modified environments in Sri Lanka. International Journal of Primatology, 33, 40-72. 